Critical exponents for groups of isometries

Sharp, Richard

2007

MIMS EPrint: 2007.181

Manchester Institute for Mathematical Sciences

School of Mathematics

The University of Manchester

\footnotetext{
Reports available from: http://eprints.maths.manchester.ac.uk/

And by contacting: The MIMS Secretary

School of Mathematics

The University of Manchester

Manchester, M13 9PL, UK
}

ISSN 1749-9097 


\title{
Critical exponents for groups of isometries
}

\author{
Richard Sharp
}

Received: 16 August 2006 / Accepted: 15 February 2007 / Published online: 29 March 2007

(C) Springer Science+Business Media B.V. 2007

\begin{abstract}
Let $\Gamma$ be a convex co-compact group of isometries of a CAT $(-1)$ space $X$ and let $\Gamma_{0}$ be a normal subgroup of $\Gamma$. We show that, provided $\Gamma$ is a free group, a sufficient condition for $\Gamma$ and $\Gamma_{0}$ to have the same critical exponent is that $\Gamma / \Gamma_{0}$ is amenable.
\end{abstract}

Keywords CAT(-1)space · Riemannian manifold · Negative curvature - Group of isometrics $\cdot$ Critical exponent $\cdot$ Amenable group

Mathematics Subject Classifications (2000) 20F67 · 20F69 - 37C35 - 60B99

\section{Introduction and results}

Let $\Gamma$ be a group of isometries acting freely and properly discontinuously on a CAT(-1) space $X$. Roughly speaking, a CAT(-1) space is a path metric space for which every geodesic triangle is more pinched than a congruent triangle in the hyperbolic plane; see [5] for a formal definition. Prototypical examples of CAT(-1) spaces are simply connected Riemannian manifold with sectional curvatures bounded above by -1 and (simplicial or non-simplicial) $\mathbb{R}$-trees.

A fundamental quantity associated to $\Gamma$ is its critical exponent $\delta(\Gamma)$. This is defined to be the abscissa of convergence of the Poincaré series

$$
\wp_{\Gamma}(s)=\sum_{\gamma \in \Gamma} \mathrm{e}^{-s d_{X}(o, \gamma o)},
$$

R. Sharp ( $\varangle)$

University of Manchester, School of Mathematics, Oxford Road,

Manchester M13 9PL, U.K.

e-mail: sharp@maths.man.ac.uk 
where $o \in X$ and $d_{X}(\cdot, \cdot)$ denotes the distance in $X$. In other words, the series converges for $s>\delta(\Gamma)$ and diverges for $s<\delta(\Gamma)$. An equivalent definition is that

$$
\delta(\Gamma)=\limsup _{T \rightarrow+\infty} \frac{1}{T} \log \#\left\{\gamma \in \Gamma: d_{X}(o, \gamma o) \leq T\right\} .
$$

A simple calculation shows that $\delta(\Gamma)$ is independent of the choice of $x \in X$.

Let $\partial X$ denote the ideal boundary of $X$. The set $\{\gamma o: \gamma \in \Gamma\}$ accumulates on a subset $\Lambda_{\Gamma} \subset \partial X$ (independent of $o$ ) called the limit set of $\Gamma$. Let $\mathcal{C}_{\Gamma}=$ c.h. $\left(\Lambda_{\Gamma}\right) \cap \mathcal{X}$, where c.h. $\left(\Lambda_{\Gamma}\right)$ is the geodesic convex hull of $\Lambda_{\Gamma}$. We say that $\Gamma$ is convex co-compact if $\mathcal{C}_{\Gamma} / \Gamma$ is compact. (If $\Gamma$ is a Kleinian group, this agrees with the classical notion of convex co-compactness.) In addition, we say that $\Gamma$ is non-elementary if it is not a finite extension of a cyclic group. These two conditions ensure that $\delta(\Gamma)>0$ and the limit in (1.2) exists.

Now suppose that $\Gamma_{0}$ is a normal subgroup of a convex co-compact group $\Gamma$. Then $\Gamma_{0}$ itself has a critical exponent $\delta\left(\Gamma_{0}\right)$ and, clearly, $\delta\left(\Gamma_{0}\right) \leq \delta(\Gamma)$. Our main result addresses the question of when we have equality.

Theorem 1 If $\Gamma / \Gamma_{0}$ is amenable then $\delta\left(\Gamma_{0}\right)=\delta(\Gamma)$.

The definition of amenable group is given in the next section.

Remark Equality of $\delta\left(\Gamma_{0}\right)$ and $\delta(\Gamma)$ was previously known to hold when $\Gamma / \Gamma_{0}$ is finite or abelian [15]. (In fact, the results in ref. [15] are stated in the case where $X$ is real hyperbolic space but the proofs given there apply more generally.)

Since obtaining the results in this paper, we have learned that Theorem 1 has been proved by Roblin [16], without the restriction that $\Gamma$ is a free group, using completely different methods. However, we feel that our alternative approach, based on approximating $\delta(\Gamma)$ and $\delta\left(\Gamma_{0}\right)$ by quantities related to random walks on graphs, has independent interest. It is worth remarking that the equality of the two critical exponents has been used recently in ref. [10].

We shall now outline the contents of the paper. In Sect. 1, we give definition of amenable groups and introduce Grigorchuk's co-growth criterion, interpreting it in terms of a graph. In Sect. 2, we describe how to write the Poincaré series $\wp_{\Gamma}(s)$ and $\wp \Gamma_{0}(s)$ in terms of a subshift of finite type. We also introduce sequences of matrices which are used to approximate $\delta(\Gamma)$ and $\delta\left(\Gamma_{0}\right)$. In Sect. 3, we use ideas from the theory of random walks on graphs, in particular [12], to show that, if $\Gamma / \Gamma_{0}$ is amenable then the respective approximations to $\delta(\Gamma)$ and $\delta\left(\Gamma_{0}\right)$ agree at each stage, from which Theorem 1 follows. In the final section, we consider that special case of $X=\mathbb{H}^{n+1}$.

I am very grateful to the referee for suggesting numerous improvements to the exposition.

\section{Amenable groups and co-growth}

Amenable groups were defined by von Neumann. A group $G$ is said to be amenable if there is an invariant mean on $L^{\infty}(G, \mathbb{R})$, i.e. a bounded linear functional $\mu: L^{\infty}(G, \mathbb{R}) \rightarrow \mathbb{R}$ such that, for any $f \in L^{\infty}(G, \mathbb{R})$,

(1) $\inf _{g \in G} f(g) \leq \mu(f) \leq \sup _{g \in G} f(g)$; and

(2) for all $g \in G, \mu(g \cdot f)=\mu(f)$, where $g \cdot f(x)=f\left(g^{-1} x\right)$.

Springer 
It is immediate from the definition that any finite group is amenable by setting

$$
\mu(f)=\frac{1}{|G|} \sum_{g \in G} f(g) .
$$

The situation for infinite groups is more subtle and we shall restrict our discussion to finitely generated groups.

A group with subexponential growth is amenable [2,7]. In particular, any abelian or nilpotent group is amenable. However, there are examples of amenable groups with exponential growth (e.g. the lamplighter groups [8]). In contrast, non-abelian free groups and, more generally, non-elementary Gromov hyperbolic groups are not amenable. It was conjectured by von Neumann that a group fails to be amenable only if it contains the free group on two generators; however, a counterexample to this was constructed by Ol'shanskii [11].

Grigorchuk related amenability to the property of co-growth of subgroups of free groups. Let $\Gamma$ (considered as an abstract group) be the free group on $k$ generators $\left\{a_{1}, \ldots, a_{k}\right\}$ and let $|\gamma|$ denote the word length of $\gamma$, i.e. the length of the shortest representation of $\gamma$ as a word in $a_{1}^{ \pm 1}, \ldots, a_{k}^{ \pm 1}$. Clearly, we have that

$$
\lim _{n \rightarrow+\infty}(\#\{\gamma \in \Gamma:|g|=n\})^{1 / n}=2 k-1 .
$$

Now suppose that $\Gamma_{0}$ is a normal subgroup of $\Gamma$. Grigorchuk showed that the co-growth $c\left(\Gamma_{0}\right)$, defined by

$$
c\left(\Gamma_{0}\right):=\limsup _{n \rightarrow+\infty}\left(\#\left\{g \in \Gamma_{0}:|g|=n\right\}\right)^{1 / n}
$$

is equal to $2 k-1$ if and only if $G=\Gamma / \Gamma_{0}$ is amenable [6] (see also [4]).

Grigorchuk's result may be reinterpreted in terms of graphs. Let $\mathcal{G}$ denote the graph consisting of one vertex and $k$ oriented edges, labelled by $a_{1}, \ldots, a_{k}$. The same edges with the reverse orientation will be labelled $a_{1}^{-1}, \ldots, a_{k}^{-1}$, respectively. Write $\mathcal{T}$ for the universal cover of $\mathcal{G}$; then $\mathcal{T}$ is a $2 k$-regular tree. It is an easy observation that $\Gamma$ acts freely on $\mathcal{T}$ with quotient $\mathcal{G}$. Furthermore, we may identify elements of word length $n$ in $\Gamma$ with non-backtracking paths of length $n$ in $\mathcal{G}$. (A path $\left(e_{1}, \ldots, e_{n}\right)$ is said to be non-backtracking if, for each $i=2, \ldots, n$, the edge $e_{i}$ is not equal to $e_{i-1}$ with the reversed orientation.)

Now consider the action of the subgroup $\Gamma_{0}$ on $\mathcal{T}$ and write $\widetilde{\mathcal{G}}=\mathcal{T} / \Gamma_{0}$, for the quotient graph; this is a $G$-cover of $\mathcal{G}$. (In fact, $\widetilde{\mathcal{G}}$ is the Cayley graph of $G$ with respect to the generators obtained from $a_{1}, \ldots, a_{k}$.) Then we may identify elements of word length $n$ in $\Gamma_{0}$ with non-backtracking paths of length $n$ in $\widetilde{\mathcal{G}}$ starting from and ending at some fixed vertex. Grigorchuk's result may then be reformulated as saying that the growth rate of the number of paths of length $n$ in $\widetilde{\mathcal{G}}$, starting from and ending at a fixed vertex, is equal to the corresponding growth rate for paths in $\mathcal{G}$ if and only if $\Gamma / \Gamma_{0}$ is amenable.

The parallels between equality of these growth rates and equality of the critical exponents is apparent. However, the "lengths" are different: word length $|\gamma|$ in one setting and the displacement $d(o, \gamma o)$ for the action on $X$ in the other. Nevertheless, this will provide the basis for our approach. In this context, we note that there exists $A>1$ such that

$$
A^{-1}|\gamma| \leq d(o, \gamma o) \leq A|\gamma| .
$$


We shall use several properties of the graph $\widetilde{\mathcal{G}}$. Firstly, provided it is not itself a tree (which only occurs if $\Gamma_{0}$ is trivial) $\widetilde{\mathcal{G}}$ has the property that "small cycles are dense" [12]: there exists $R>0$ such that, for each vertex $u$ in $\widetilde{\mathcal{G}}$, the set $B(u, R)=\left\{v: d_{\mathcal{G}}(u, v) \leq R\right\}$ contains a cycle. We also note that there is a number $L(R)>0$ such that, for every vertex $u$ in $\widetilde{\mathcal{G}}, \# B(u, R) \leq L(R)$.

Later we shall need to find paths joining vertices in $\widetilde{\mathcal{G}}$. Let $c_{n}(u, v)$ denote the number of non-backtracking paths of length $n$ in $\widetilde{\mathcal{G}}$ from $u$ to $v$.

Lemma 2.1 [17] Let $u, v$ be vertices of $\widetilde{\mathcal{G}}$. Then either

$$
\lim _{n \rightarrow+\infty} c_{n}(u, v)^{1 / n}=c\left(\Gamma_{0}\right)
$$

or

$$
\lim _{n \rightarrow+\infty} c_{2 n+\delta(u, v)}(u, v)^{1 / 2 n}=c\left(\Gamma_{0}\right) \text { and } c_{2 n+\delta(u, v)-1}(u, v)=0,
$$

where $\delta(u, v)=0$ if $d_{\mathcal{G}}(u, v)$ is even and $\delta(u, v)=1$ if $d_{\mathcal{G}}(u, v)$ is odd.

Corollary 2.1.1 Suppose that $G$ is amenable (or even that $c\left(\Gamma_{0}\right)>0$ ) and let $u, v$ be vertices of $\widetilde{\mathcal{G}}$. Then there exists $l(u, v)>0$ such that either $c_{l(u, v)}(u, v)>0$ or $c_{l(u, v)-1}(u, v)>0$.

\section{Shifts of Finite Type and Approximation}

Recall that the free group $\Gamma$ is given in terms of generators $\mathcal{A}=\left\{a_{1}^{ \pm 1}, \ldots, a_{k}^{ \pm 1}\right\}$. We shall form a subshift of finite type $\sigma: \Sigma \rightarrow \Sigma$, where

$$
\Sigma=\left\{x=\left(x_{i}\right)_{i=0}^{\infty} \in \mathcal{A}^{\mathbb{Z}^{+}}: x_{i+1} \neq x_{i}^{-1}, \forall i \in \mathbb{Z}^{+}\right\}
$$

and $\sigma$ is the shift map: $(\sigma x)_{i}=x_{i+1}$. We call $\left(x_{0}, \ldots, x_{n-1}\right) \in \mathcal{A}^{n}$ an allowed string of length $n$ if $x_{i+1} \neq x_{i}^{-1}, i=0, \ldots, n-2$. We write $\Sigma_{n}$ for the set of all allowed strings of length $n, \Sigma_{\leq n}=\bigcup_{m=0}^{n} \Sigma_{m}$ and $\Sigma^{*}=\bigcup_{n=0}^{\infty} \Sigma_{n}$, where $\Sigma_{0}$ is defined to be a singleton consisting of an "empty string" $\omega$. There is an obvious bijection between $\Sigma_{n}$ and elements of $\Gamma$ with word length $n$ (and hence between $\Gamma$ and $\Sigma^{*}$ ).

We make $\Sigma \cup \Sigma^{*}$ into a metric space by setting $d(x, y)=2^{-n(x, y)}$, where

$$
n(x, y)=\left\{\begin{array}{l}
0, \quad \text { if } x_{0} \neq y_{0}, \\
\sup \left\{n \geq 0: x_{m}=y_{m}, 0 \leq m \leq n\right\}, \quad \text { otherwise }
\end{array}\right.
$$

If $f: \Sigma \cup \Sigma^{*} \rightarrow \mathbb{R}$ is Hölder continuous with Hölder exponent $\alpha>0$ then we write

$$
|f|_{\alpha}=\sup \left\{\frac{f(x)-f(y)}{d(x, y)^{\alpha}}: x \neq y\right\} .
$$

If we define $\sigma(\omega)=\omega$, the shift map extends to $\sigma: \Sigma \cup \Sigma^{*} \rightarrow \Sigma \cup \Sigma^{*}$ and $\sigma\left(\Sigma_{n}\right)=$ $\Sigma_{n-1}, n \geq 1$. For a function $f: \Sigma \cup \Sigma^{*} \rightarrow \mathbb{R}$, we write $f^{n}(x)=f(x)+f(\sigma x)+\cdots+$ $f\left(\sigma^{n-1} x\right)$.

Proposition 3.1 $[9,13,14]$ There is a strictly positive Hölder continuous function $r$ : $\Sigma \cup \Sigma^{*} \rightarrow \mathbb{R}$ such that, if $\gamma=x_{0} \cdots x_{n-1}$ then

$$
r^{n}\left(x_{0}, \ldots, x_{n-1}\right)=d_{X}(o, \gamma o) \text {. }
$$


Remark An examination of the proof in ref. [14] shows that what is essential for the proof is that $X$ satisfies the Aleksandrov-Toponogov Comparison property. Thus, the result holds if $X$ is a CAT( $(-1)$ space.

An easy calculation then shows that

$$
\wp_{\Gamma}(s)=1+\sum_{n=1}^{\infty} \sum_{x \in \sigma^{-n}(\omega) \backslash\{\omega\}} \mathrm{e}^{-s r^{n}(x)} .
$$

Let $\psi: \Gamma \rightarrow G=\Gamma / \Gamma_{0}$ be the natural homomorphism and, for $x=\left(x_{0}, \ldots, x_{n-1}\right) \in$ $\Sigma_{n}$, write $\psi_{n}(x)=\psi\left(x_{0}\right) \cdots \psi\left(x_{n-1}\right)$. We have

$$
\wp \Gamma_{0}(s)=1+\sum_{n=1}^{\infty} \sum_{\substack{x \in \sigma^{-n}(\omega) \backslash\{\omega\} \\ \psi_{n}(x)=e}} \mathrm{e}^{-s r^{n}(x)} .
$$

We shall study the abscissas of convergence of the above two series via a sequence of approximations to $r$. We define

$$
r_{N}(x)=\left\{\begin{array}{l}
r(x), \quad \text { if } x \in \Sigma_{n}, n \leq N \\
r\left(x_{0}, \ldots, x_{N-1}\right), \quad \text { otherwise. }
\end{array}\right.
$$

Then $\left\|r-r_{N}\right\|_{\infty} \leq|r|_{\alpha} 2^{-\alpha(N+1)}$, where $\alpha>0$ is the Hölder exponent of $r$. Hence, given $\epsilon>0$, we can choose $N$ sufficiently large so that, for each $x \in \Sigma \cup \Sigma^{*}$ and $n \geq 1$, $\left|r^{n}(x)-r_{N}^{n}(x)\right|<n \epsilon$.

We define $\delta_{N}$ and $\delta_{N}^{0}$ to be the abscissas of convergence of $\wp_{N}(s)$ and $\wp_{N}^{0}(s)$, respectively, where

$$
\wp_{N}(s)=1+\sum_{n=1}^{\infty} \sum_{x \in \sigma^{-n}(\omega) \backslash\{\omega\}} \mathrm{e}^{-s r_{N}^{n}(x)}, \wp_{N}^{0}(s)=1+\sum_{n=1}^{\infty} \sum_{\substack{x \in \sigma^{-n}(\omega) \backslash\{\omega\} \\ \psi_{n}(x)=e}} \mathrm{e}^{-s r_{N}^{n}(x)} .
$$

Lemma 3.1 We have $\lim _{N \rightarrow+\infty} \delta_{N}=\delta(\Gamma)$ and $\lim _{N \rightarrow+\infty} \delta_{N}^{0}=\delta\left(\Gamma_{0}\right)$.

Proof For $\gamma=x_{0} \cdots x_{|\gamma|-1} \in \Gamma$, let $x_{\gamma}=\left(x_{0}, \ldots, x_{|\gamma|-1}\right) \in \Sigma^{*}$. Then, $r^{|\gamma|}\left(x_{\gamma}\right)=$ $d(o, \gamma o)$, so, using this notation,

$$
\delta(\Gamma)=\limsup _{T \rightarrow+\infty} \frac{1}{T} \log \#\left\{\gamma: r^{|\gamma|}\left(x_{\gamma}\right) \leq T\right\}, \delta_{N}=\limsup _{T \rightarrow+\infty} \frac{1}{T} \log \#\left\{\gamma: r_{N}^{|\gamma|}\left(x_{\gamma}\right) \leq T\right\} .
$$

Fix $\epsilon>0$ sufficiently small that $A \epsilon<1$, where $A$ is given by (2.1). Then, provided $N$ is sufficiently large, $r^{|\gamma|}\left(x_{\gamma}\right) \leq r_{N}^{|\gamma|}\left(x_{\gamma}\right)+|\gamma| \epsilon \leq r_{N}^{|\gamma|}\left(x_{\gamma}\right)+A r^{|\gamma|}\left(x_{\gamma}\right) \epsilon$ and so

$$
r^{|\gamma|}\left(x_{\gamma}\right) \leq \frac{r_{N}^{|\gamma|}\left(x_{\gamma}\right)}{1-A \epsilon} \text {. }
$$

Hence

$$
\#\left\{\gamma: r_{N}^{|\gamma|}\left(x_{\gamma}\right) \leq T\right\} \leq \#\left\{\gamma: r^{|\gamma|}\left(x_{\gamma}\right) \leq(1-A \epsilon)^{-1} T\right\}
$$

and so $\delta_{N} \leq(1-A \epsilon)^{-1} \delta(\Gamma)$. Since we may take $\epsilon$ arbitrarily small, we conclude that $\lim \sup _{N \rightarrow+\infty} \delta_{N} \leq \delta(\Gamma)$. A similar argument gives the corresponding lower bound, so we have $\lim _{N \rightarrow+\infty} \delta_{N}=\delta(\Gamma)$. The same proof gives the result for $\delta_{N}^{0}$. 
Hence, to prove Theorem 1, it suffices to show that if $G$ is amenable then $\delta_{N}=\delta_{N}^{0}$, for each $N \geq 1$. We shall do this in the next section. First we need to rewrite $\wp_{N}(s)$ and $\wp_{N}^{0}(s)$ in matrix form.

For $N \geq 1$, define matrices $P_{N}$, indexed by $\Sigma_{N} \times \Sigma_{N}$, by

$$
P_{N}(x, y)=\left\{\begin{array}{l}
\mathrm{e}^{-\delta_{N} r_{N}\left(x_{0}, x_{1}, \ldots, x_{N-1}, y_{N-1}\right)}, \quad \text { if } x_{n}=y_{n-1}, \quad n=1, \ldots, N-1, \\
0, \quad \text { otherwise },
\end{array}\right.
$$

where $x=\left(x_{0}, x_{1}, \ldots, x_{N-1}\right), y=\left(y_{0}, y_{1}, \ldots, y_{N-1}\right)$. (For $N=1$, we set $P_{1}\left(x_{0}, y_{0}\right)=0$ whenever $y_{0}=x_{0}^{-1}$. For $N \geq 2$ this is automatically avoided.) Each $P_{N}$ is irreducible (and aperiodic). Also define another sequence of matrices $Q_{N}$, indexed by $\Sigma_{\leq N} \times \Sigma_{\leq N}$, by

$$
Q_{N}(x, y)=\left\{\begin{array}{l}
\mathrm{e}^{-\delta_{N} r_{N}\left(x_{0}, x_{1}, \ldots, x_{N-1}, y_{N-1}\right)}, \quad \text { if } x_{n}=y_{n-1}, \quad n=1, \ldots, N-1 \\
0, \quad \text { otherwise }
\end{array}\right.
$$

where, for $x \in \Sigma_{m}$, we write $x=(x_{0}, \ldots, x_{m-1}, \underbrace{\omega, \ldots, \omega}_{N-m})$. The matrices $Q_{N}$ are not irreducible. Note that $P_{N}$ is the restriction of $Q_{N}$ to $\Sigma_{N} \times \Sigma_{N}$.

From the definition of $Q_{N}$, we have that, for $n>N$,

$$
\sum_{x \in \sigma^{-n}(\omega) \backslash\{\omega\}} \mathrm{e}^{-\delta_{N} r_{N}^{n}(x)}=\sum_{x \in \Sigma_{N}} \sum_{a \in \Sigma_{1}} Q_{N}^{n}(x,(a, \omega, \ldots, \omega)) .
$$

Now, since $P_{N}$ is irreducible, the value of $\lim \sup _{n \rightarrow+\infty}\left(P_{N}^{n}(x, y)\right)^{1 / n}$ is independent of $x, y \in \Sigma_{N}$ (in fact it is the spectral radius of $P_{N}$ ).

Lemma 3.2 For any $x, y \in \Sigma_{N}$ and $a \in \Sigma_{1}$,

$$
\limsup _{n \rightarrow+\infty}\left(P_{N}^{n}(x, y)\right)^{1 / n}=\limsup _{n \rightarrow+\infty}\left(Q_{N}^{n}(x,(z, \omega, \ldots, \omega))\right)^{1 / n} .
$$

Proof We have

$$
\begin{aligned}
Q_{N}^{n}(x,(a, \omega, \ldots, \omega)) & =\sum_{y \in \Sigma_{N}} Q_{N}^{n-N}(x, y) Q_{N}^{N}(y,(a, \omega, \ldots, \omega)) \\
& =\sum_{y \in \Sigma_{N}} P_{N}^{n-N}(x, y) Q_{N}^{N}(y,(a, \omega, \ldots, \omega)) .
\end{aligned}
$$

Since $\delta_{N}$ is the abscissa of convergence of $\wp_{N}(s)$, we deduce that, for each $x, y \in \Sigma_{N}$, $\lim \sup _{n \rightarrow+\infty}\left(P_{N}^{n}(x, y)\right)^{1 / n}=1$.

By the Perron-Frobenius Theorem, $P_{N}$ has 1 as an eigenvalue and an associated strictly positive (row) eigenvector $v_{N}: v_{N} P_{N}=v_{N}$. In addition, we may suppose that $P_{N}$ is normalized so that

$$
\sum_{y \in \Sigma_{N}} P_{N}(x, y)=1
$$

In other words, $P_{N}$ may be regarded as a matrix of transition probabilities between elements of $\Sigma_{N}$. 
Now we define another sequence of (infinite) matrices $\widetilde{P}_{N}, N \geq 1$, indexed by $\left(\Sigma_{N} \times G\right) \times\left(\Sigma_{N} \times G\right)$, by

$$
\widetilde{P}_{N}((x, g),(y, h))=\left\{\begin{array}{l}
P_{N}(x, y), \quad \text { if } \psi\left(x_{0}\right)=g^{-1} h, \\
0, \quad \text { otherwise. }
\end{array}\right.
$$

(Note that the exponent in the entries of $\widetilde{P}_{N}$ is $\delta_{N}$ not $\delta_{N}^{0}$.) Each $\widetilde{P}_{N}$ is locally finite in the sense that, for each $(x, g)$, there are only finitely many $(y, h)$ such that $\widetilde{P}_{N}((x, g),(y, h))>0$.

We also define a corresponding sequence of infinite matrices $\widetilde{Q}_{N}, N \geq 1$, indexed by $\left(\Sigma_{\leq N} \times G\right) \times\left(\Sigma_{\leq N} \times G\right)$, by

$$
\widetilde{Q}_{N}((x, g),(y, h))=\left\{\begin{array}{l}
Q_{N}(x, y), \quad \text { if } \psi\left(x_{0}\right)=g^{-1} h, \\
0, \quad \text { otherwise }
\end{array}\right.
$$

We have

$$
\sum_{\substack{x \in \sigma^{-n}(\omega) \backslash\{\omega\} \\ \psi_{n}(x)=e}} \mathrm{e}^{-s r_{N}^{n}(x)}=\sum_{x \in \Sigma_{N}} \sum_{y \in \Sigma_{1}} \widetilde{Q}_{N}^{n}((x, e),((y, \omega, \ldots, \omega), e)) .
$$

In Sect. 4, we shall prove the following lemma.

Lemma 3.3 $G$ is amenable if and only if $\lim \sup _{n \rightarrow+\infty}\left(\widetilde{P}_{N}^{n}((x, e),(y, e))\right)^{1 / n}=1$.

This lemma implies that, provided $G$ is amenable, $\delta_{N}=\delta_{N}^{0}, N \geq 1$. Combining this with Lemma 2.1 gives Theorem 1.

\section{An Auxiliary Estimate}

In this section, we establish an estimate needed to complete the proof of Lemma 2.3 in Sect. 4.

Write Fix $_{n}=\left\{x \in \Sigma: \sigma^{n} x=x\right\}$. If $x=\left(x_{0}, x_{1}, \ldots, x_{n-1}, x_{0}, \ldots\right) \in$ Fix $_{n}$, write $x^{-1}=\left(x_{n-1}^{-1}, \ldots, x_{1}^{-1}, x_{0}^{-1}, x_{n-1}^{-1}, \ldots\right) \in \operatorname{Fix}_{n}$.

Lemma 4.1 For each $N \geq 1, r_{N}^{n}(x)=r_{N}^{n}\left(x^{-1}\right)$ whenever $x \in$ Fix $_{n}, n \geq 1$.

Proof For $n \geq N$,

$$
\begin{aligned}
r_{N}^{n}(x)= & r\left(x_{0}, x_{1}, \ldots, x_{N-1}\right)+r\left(x_{1}, x_{2}, \ldots, x_{N}\right)+\cdots+r\left(x_{n-1}, x_{0}, \ldots, x_{N-2}\right) \\
= & d\left(o, x_{0} x_{1} \cdots x_{N-1} o\right)-d\left(o, x_{1} \cdots x_{N-1} o\right) \\
& +d\left(o, x_{1} x_{2} \cdots x_{N} o\right)-d\left(o, x_{2} \cdots x_{N} o\right) \\
& +\cdots+d\left(o, x_{n-1} x_{0} \cdots x_{N-2} o\right)-d\left(o, x_{0} \cdots x_{N-2} o\right) .
\end{aligned}
$$

On the other hand, 


$$
\begin{aligned}
r_{N}^{n}\left(x^{-1}\right)= & r\left(x_{n-1}^{-1}, x_{n-2}^{-1}, \ldots, x_{n-N}^{-1}\right)+r\left(x_{n-2}^{-1}, x_{n-3}^{-1}, \ldots, x_{n-N-1}^{-1}\right) \\
& +\cdots+r\left(x_{0}^{-1}, x_{n-1}^{-1}, \ldots, x_{n-N+1}^{-1}\right) \\
= & d\left(o, x_{n-1}^{-1} x_{n-2}^{-1} \cdots x_{n-N}^{-1} o\right)-d\left(o, x_{n-2}^{-1} \cdots x_{n-N}^{-1} o\right) \\
& +d\left(o, x_{n-2}^{-1} x_{n-3}^{-1} \cdots x_{n-N-1}^{-1} o\right)-d\left(o, x_{n-3}^{-1} \cdots x_{n-N-1}^{-1} o\right) \\
& +\cdots+d\left(o, x_{0}^{-1} x_{n-1}^{-1} \cdots x_{n-N+1}^{-1} o\right)-d\left(o, x_{n-1}^{-1} \cdots x_{n-N+1}^{-1} o\right) \\
= & d\left(o, x_{n-N} \cdots x_{n-2} x_{n-1} o\right)-d\left(o, x_{n-N} \cdots x_{n-2} o\right) \\
& +d\left(o, x_{n-N-1} \cdots x_{n-3} x_{n-2} o\right)-d\left(o, x_{n-N-1} \cdots x_{n-3} o\right) \\
& +\cdots+d\left(o, x_{n-N+1} \cdots x_{n-1} x_{0} o\right)-d\left(o, x_{n-N+1} \cdots x_{n-1} o\right)=r_{N}^{n}(x) .
\end{aligned}
$$

If $n<N$, the calculations become easier.

Consider the restriction $r_{N}: \Sigma_{N} \rightarrow \mathbb{R}$. We can define another function $\check{r}_{N}: \Sigma_{N} \rightarrow \mathbb{R}$ by $\check{r}_{N}\left(x_{0}, \ldots, x_{N-1}\right)=r_{N}\left(x_{N-1}^{-1}, \ldots, x_{0}^{-1}\right)$. Applying Livsic's theorem for finite directed graphs to the above result, we may deduce:

Corollary 4.1.1 There exists $u: \Sigma_{N-1} \rightarrow \mathbb{R}$ such that

$$
r_{N}\left(x_{0}, x_{1}, \ldots, x_{N-1}\right)=r_{N}\left(x_{N-1}^{-1}, \ldots, x_{1}^{-1}, x_{0}^{-1}\right)+u\left(x_{1}, \ldots, x_{N-1}\right)-u\left(x_{0}, \ldots, x_{N-2}\right) .
$$

Lemma 4.2 There exists a constant $C_{0}>0$ such that, for all $(x, g),(y, h) \in \Sigma_{N} \times G$ and $n \geq 1$,

$$
P_{N}^{n}((x, g),(y, h)) \leq C_{0} P_{N}^{n}\left(\left(\check{y}, h^{-1}\right),\left(\check{x}, g^{-1}\right)\right),
$$

where, if $x=\left(x_{0}, x_{1}, \ldots, x_{N-1}\right)$ and $y=\left(y_{0}, y_{1}, \ldots, y_{N-1}\right)$, we use the notation $\breve{x}=$ $\left(x_{N-1}^{-1}, \ldots, x_{1}^{-1}, x_{0}^{-1}\right)$ and $\check{y}=\left(y_{N-1}^{-1}, \ldots, y_{1}^{-1}, y_{0}^{-1}\right)$.

We may take

$$
C_{0}=\exp \left(2 \delta_{N} \sup \left\{|u(x)|: x \in \Sigma_{N-1}\right\}\right)
$$

\section{Random Walks on Graphs}

In order to prove Lemma 3.3, we shall adapt work of Ortner and Woess on non-backtracking random walks on graphs contained in ref. [12].

For each $N \geq 1$, we define an (undirected) graph $\mathcal{S}_{N}$ with vertex set $\Sigma_{N} \times G$. Two vertices $(x, g)$ and $(y, h)$ will be joined by an edge if and only if either $\widetilde{P}_{N}((x, g),(y, h))>$ 0 or $\widetilde{P}_{N}((y, h),(x, g))>0$. We note that $\mathcal{S}_{N}$ is connected and that each vertex has degree $2 k$.

We may think of $\widetilde{P}_{N}$ as defining a Markov process on $\mathcal{S}_{N}$. As part of the proof of Lemma 3.3, we will show that $\widetilde{P}_{N}$ has the following three properties [12]:

(1) $\widetilde{P}_{N}$ has bounded range, i.e. there exists $R>0$ such that if $\widetilde{P}_{N}((x, g),(y, h))>0$ then $(x, g)$ and $(y, h)$ are at distance $\leq R$ in $\mathcal{S}_{N}$.

(2) $\widetilde{P}_{N}$ has a bounded invariant measure; i.e. there exists a function $v: \Sigma_{N} \times G \rightarrow \mathbb{R}^{+}$, bounded above and below away from zero, such that, for all $(y, h) \in \Sigma_{N} \times G$,

$$
\sum_{(x, g) \in \Sigma_{N} \times G} \widetilde{P}_{N}((x, g),(y, h)) v((x, g))=v((y, h)) .
$$


(3) $\widetilde{P}_{N}$ is uniformly irreducible, i.e. there exist constants $K>0, \epsilon>0$ such that, for any pair of neighbouring vertices $(x, g),(y, h)$ in $\mathcal{S}_{N}$, one can find $k \leq K$ such that $\widetilde{P}_{N}^{k}((x, g),(y, h)) \geq \epsilon$.

We note that (1) holds immediately with $R=1$.

To show (2), let recall that there is a strictly positive row vector $v_{N}=\left(v_{N}(x)\right)_{x \in \Sigma_{N}}$ such that $v_{N} P_{N}=v_{N}$. Define $v$ by $v((x, g))=v_{N}(x)$. Clearly this is bounded above and below away from zero. A simple calculation shows it has the desired $\widetilde{P}_{N}$-invariance.

Finally, we show that $\widetilde{P}_{N}$ is uniformly irreducible.

\section{Lemma 5.1 $\widetilde{P}_{N}$ is uniformly irreducible.}

Proof Fix a number $K$ (to be determined later). Let $\epsilon_{0}<1$ denote the smallest positive entry of $\widetilde{P}_{N}$ and let $\epsilon=\epsilon_{0}^{K}$; then, for every $k \leq K$, each positive entry of $\widetilde{P}_{N}^{k}$ is greater than or equal to $\epsilon$. Let $(x, g)$ and $(y, h)$ be neighbouring vertices in $\mathcal{S}_{N}$. Without lose of generality, $\widetilde{P}_{N}((x, g),(y, h))>\epsilon$ and $\widetilde{P}_{N}((y, h),(x, g))=0$. To complete the proof we need to find a positive probability path of length at most $K$ from $(y, h)$ to $(x, g)$.

Observe that we can identify $\Sigma_{N} \times G$ with the set of non-backtracking paths of length $N$ in $\widetilde{\mathcal{G}}$ and a positive probability path of length $k$ in $\mathcal{S}_{N}$ corresponds to a non-backtracking path of length $N+k$ in $\widetilde{\mathcal{G}}$. We therefore need to show that, for any two non-backtracking paths (given by sequences of vertices) $\left(u_{0}, u_{1}, \ldots, u_{N}\right)$ and $\left(v_{0}, v_{1}, \ldots, v_{N}\right)$ in $\widetilde{\mathcal{G}}$, there exists $k \leq K$ such that there is a non-backtracking path of length $k$ joining them to give a non-backtracking path from $u_{0}$ to $v_{N}$. It follows from Corollary 2.1.1 that there is a non-backtracking path $\left(u_{N}, w_{1}, \ldots, w_{\kappa-1}, v_{0}\right)$, with $\kappa \leq l\left(u_{N}, v_{0}\right)$, joining $u_{N}$ to $v_{0}$. However, it is possible then when this is inserted between the other two paths, backtracking occurs. To avoid this we shall use the "small cycles are dense" property of $\widetilde{\mathcal{G}}$. (The following part of the proof is adapted from the proof of Lemma 4.7 in ref. [12].)

First, we consider the beginning of the inserted path. If $w_{1} \neq u_{N-1}$ there is nothing to do, so suppose that $w_{1}=u_{N-1}$. Choose a neighbour $z_{1}$ of $u_{N}$ which is not equal to $u_{N-1}$. By Lemma 4.3 of [12], $\left(u_{N}, z_{1}\right)$ may be extended into non-backtracking paths which reach infinitely many vertices. Since $B\left(u_{N-1}, R\right)$ is finite, we may choose one of these paths, $\left(u_{N}, z_{1}, \ldots, z_{r}\right)$, so that $z_{r} \notin B\left(u_{N-1}, R\right)$ but $z_{i} \in B\left(u_{N-1}, R\right)$, $i=1, \ldots, r-1$ (with $r \leq L(R)+1$. By the "small cycles are dense" property, there is a cycle $\left(c_{0}, c_{1}, \ldots, c_{p-1}, c_{0}\right)$ in $B\left(z_{r}, R\right)$ (with $\left.p \leq L(R)\right)$. Either

(a) $z_{r}=c_{i}$ for some $i=0,1, \ldots, p-1$, or,

(b) by the definition of $B\left(z_{r}, R\right)$, there is a non-backtracking path $\left(z_{r}, a_{1}, \ldots, a_{q-1}, c_{0}\right)$ $\left(a_{1} \neq z_{r-1}\right)$ joining $z_{r}$ to $c_{0}$ (with $q \leq R$ ).

In case (a), we insert

$$
\left(u_{N}, z_{1}, \ldots, z_{r}, c_{i+1}, \ldots, c_{p-1}, c_{0}, \ldots, c_{i-1}, z_{r}, z_{r-1}, \ldots, z_{1}, u_{N}\right)
$$

and in case (b), we insert

$$
\left(u_{N}, z_{1}, \ldots, z_{r}, a_{1}, \ldots, a_{q-1}, c_{0}, c_{1}, \ldots, c_{p-1}, c_{0}, a_{q-1}, \ldots, a_{1}, z_{r}, z_{r-1}, \ldots, z_{1}, u_{N}\right)
$$

between $\left(u_{0}, u_{1}, \ldots, u_{N}\right)$ and $\left(u_{N}, w_{1}, \ldots, w_{\kappa-1}, v_{0}\right)$.

Now consider the end of the path $\left(u_{N}, w_{1}, \ldots, w_{k-1}, v_{0}\right)$. If $w_{k-1} \neq v_{1}$ there is nothing to do. On the other hand, if $w_{k-1}=v_{1}$ then we carry out a similar construction to that in the paragraph above. 
In this way, we have obtained a non-backtracking path starting with $\left(u_{0}, u_{1}, \ldots, u_{N}\right)$ and ending with $\left(v_{0}, v_{1}, \ldots, v_{N}\right)$ with $u_{N}$ and $v_{0}$ being joined in at most $l\left(u_{N}, v_{0}\right)+$ $4(L(R)+1)+4 R+4 L(R)$ steps.

To complete the proof, we need to show that this number may be bounded independently of our initial choice of $(x, g)$ and $(y, h)$ (which determine $u_{N}$ and $\left.v_{0}\right)$. First, we note that there are only finitely many $x$ and $y$ in $\Sigma_{N}$. Second, we observe that, for any $a \in G, \widetilde{P}_{N}((x, a g),(y, a h))=\widetilde{P}_{N}((x, g),(y, h))$, so, without loss of generality, we may suppose that $g=e$. Since $(y, h)$ is a neighbour of $(x, g)$ in $\mathcal{S}_{N}$, this forces $h$ to be one of the finitely many elements $\psi\left(a_{1}^{ \pm 1}\right), \ldots, \psi\left(a_{k}^{ \pm 1}\right)$. Therefore, we may choose $K$ to be the maximum of $l\left(u_{N}, v_{0}\right)+8 L(R)+4 R+4$, taken over this finite number of choices.

Since $\widetilde{P}_{N}$ has an invariant measure $v$, it acts on the Hilbert space $l^{2}\left(\mathcal{S}_{N}, v\right)$. Let $\rho_{2}\left(\widetilde{P}_{N}\right)$ denote the spectral radius. Also, since $\widetilde{P}_{N}$ is irreducible,

$$
\rho\left(\widetilde{P}_{N}\right)=\limsup _{n \rightarrow+\infty}\left(\widetilde{P}_{N}^{n}((x, g),(y, h))\right)^{1 / n}
$$

is independent of $(x, g)$ and $(y, h)$ and $\rho\left(\widetilde{P}_{N}\right) \leq \rho_{2}\left(\widetilde{P}_{N}\right)$.

To complete the proof of Lemma 3.3 (and hence of Theorem 1) we use the following results from [12]. (See page 112 of [18] for the definition of an amenable graph.)

Proposition 5.1 [12,Theorem 4.6] If $\mathcal{S}_{N}$ is connected with bounded vertex degrees and $\widetilde{P}_{N}$ satisfies $(1)-(3)$ then $\rho_{2}\left(\widetilde{P}_{N}\right)=1$ if and only if $\mathcal{S}_{N}$ is amenable.

We have already seen that the hypotheses used in Proposition 5.1 are satisfied. The next result relates $\rho_{2}\left(\widetilde{P}_{N}\right)$ and $\rho\left(\widetilde{P}_{N}\right)$.

Proposition $5.2 \rho\left(\widetilde{P}_{N}\right)=\rho_{2}\left(\widetilde{P}_{N}\right)$.

Proof The proof is a simple modification of the proof of Proposition 2.6 in ref. [12]. The hypothesis there is that one has a graph for which "small cycles are dense"; since this holds for $\widetilde{\mathcal{G}}$, it also holds for $\mathcal{S}_{N}$. There are two differences from the proof in ref. [12]:

(1) we consider a matrix $\bar{P}_{N}=\frac{1}{2}\left(I+\widetilde{P}_{N}\right)$, where $I$ is the identity matrix, and observe that $\bar{P}_{N}$ preserves $v$ (rather than the counting measure as in ref. [12]);

(2) we use Lemma 4.2: there exists a constant $C_{0}>0$ such that, for all $(x, g),(y, h) \in$ $\Sigma_{N} \times G$ and $n \geq 1$,

$$
P_{N}^{n}((x, g),(y, h)) \leq C_{0} P_{N}^{n}\left(\left(\check{y}, h^{-1}\right),\left(\check{x}, g^{-1}\right)\right),
$$

where, if $x=\left(x_{0}, x_{1}, \ldots, x_{N-1}\right)$ and $y=\left(y_{0}, y_{1}, \ldots, y_{N-1}\right)$, we use the notation $\check{x}=\left(x_{N-1}^{-1}, \ldots, x_{1}^{-1}, x_{0}^{-1}\right)$ and $\check{y}=\left(y_{N-1}^{-1}, \ldots, y_{1}^{-1}, y_{0}^{-1}\right)$. (In ref. [12], the inequality is an equality with $C_{0}=1$.)

Neither of these affect the proof.

Together, these two results show that $\rho\left(\widetilde{P}_{N}\right)=1$ if and only if $\mathcal{S}_{N}$ is amenable. To finish things off, we show that the latter condition is equivalent to the amenability of $G$.

Recall that a map $f: X \rightarrow Y$ between two metric spaces $\left(X, d_{X}\right)$ and $\left(Y, d_{Y}\right)$ is called a quasi-isometry if there exist $A \geq 1, B, C \geq 0$ such that,

(1) for all $x, x^{\prime} \in X, A^{-1} d_{X}\left(x, x^{\prime}\right)-B \leq d_{Y}\left(f(x), f\left(x^{\prime}\right)\right) \leq A d_{X}\left(x, x^{\prime}\right)+B$; and

(2) for every $y \in Y$, there exists $x \in X$ such that $d_{Y}(y, f(x)) \leq C$.

量 Springer 
Proposition 5.3 $\mathcal{S}_{N}$ is amenable if and only if $G$ is amenable.

Proof We identify $G$ with its Cayley graph $\mathcal{C}(\mathcal{G})$; $G$ is an amenable group if and only if $\mathcal{C}(\mathcal{G})$ is an amenable graph. Define a map $f_{N}: \mathcal{S}_{N} \rightarrow \mathcal{C}(\mathcal{G})$ on the vertices by $f_{N}(x, g)=g$ and extend it to the edges by $f_{N}((x, g),(y, h))=(g, h)$. This map is clearly a quasi-isometry. Since, for graphs with bounded vertex degree, amenability is an invariant of quasi-isometry [18,Theorem 4.7], the result is proved.

\section{Kleinian Groups}

In this section, we shall discuss the relevance of our results for Kleinian groups acting on the hyperbolic space $\mathbb{H}^{n+1}$ and, in particular, for finitely generated Fuchsian results. (These results are subsumed by those in ref. [16].)

We begin be describing the results of Brooks on amenability and the spectrum of the Laplacian. Let $N$ be a complete Riemannian manifold and let $\Delta_{N}$ denote the Laplace-Beltrami operator acting on $L^{2}(N)$. Then $-\Delta_{N}$ is a positive self-adjoint operator on $L^{2}(N)$. If $\sigma\left(-\Delta_{N}\right)$ denotes the spectrum of $-\Delta_{N}$ then $\sigma\left(-\Delta_{N}\right) \subset[0,+\infty)$. Let $\lambda_{0}(N)$ denote the bottom of the spectrum, i.e.

$$
\lambda_{0}(N)=\inf \sigma\left(-\Delta_{N}\right) .
$$

If $\widetilde{N}$ is a Riemannian cover of $N$ then $\lambda_{0}(\widetilde{N}) \geq \lambda_{0}(N)$.

Theorem (Brooks [3]) Suppose that $\widetilde{N}$ is a Riemannian cover of $N$. If $\pi_{1}(N) / \pi_{1}(\widetilde{N})$ is amenable then $\lambda_{0}(\widetilde{N})=\lambda_{0}(N)$.

Remark Subject to certain conditions, in particular, if $N$ is compact, Brooks also showed the converse.

Let $\Gamma$ be a Kleinian group, i.e. a discrete group of isometries of the real $(n+1)$ dimensional hyperbolic space $\mathbb{H}^{n+1}$. We say that $\Gamma$ is geometrically finite if it is possible to choose a fundamental domain which is a finite sided polyhedron. We shall suppose that $\Gamma$ acts freely so that $\mathbb{H}^{n+1} / \Gamma$ is a smooth manifold and that $\Gamma$ is non-elementary. Then $0<\delta(\Gamma) \leq n$, with equality if and only if $\mathbb{H}^{n+1} / \Gamma$ has finite volume. As before, $\Gamma_{0}$ will be a normal subgroup of $\Gamma$.

In this setting, $\delta(\Gamma)$ is related to $\lambda_{0}\left(\mathbb{H}^{n+1} / \Gamma\right)$ by the formula

$$
\lambda_{0}\left(\mathbb{H}^{n+1} / \Gamma\right)= \begin{cases}\delta(\Gamma)(n-\delta(\Gamma)) & \text { if } \delta(\Gamma)>n / 2 \\ n^{2} / 4 & \text { if } \delta(\Gamma) \leq n / 2\end{cases}
$$

with an identical formula holding for $\Gamma_{0}$. Thus, in the range $\delta(\Gamma)>n / 2$, the critical exponent may be read off from the $\lambda_{0}$ and vice versa, while for $\delta(\Gamma) \leq n / 2$ the critical exponent is a more subtle quantity.

Using the above relation, Brooks was able to deduce that, if $\Gamma$ is geometrically finite and $\delta(\Gamma)>n / 2$ then amenability of $\Gamma / \Gamma_{0}$ implies that $\delta\left(\Gamma_{0}\right)=\delta(\Gamma)$ [3]. In the case where $\Gamma$ is a free group, we can remove the restriction that $\delta(\Gamma)>n / 2$. In particular, this gives a complete result for finitely generated Fuchsian groups.

Theorem 2 Let $\Gamma$ be a finitely generated Fuchsian group and let $\Gamma_{0}$ be a normal subgroup. If $\Gamma / \Gamma_{0}$ is amenable then $\delta\left(\Gamma_{0}\right)=\delta(\Gamma)$. 
Proof First, we note that, for Fuchsian groups, if $\Gamma$ is finitely generated then it is geometrically finite. If $\mathbb{H}^{2} / \Gamma$ is compact then $\delta(\Gamma)=1$, so Brooks's result applies. If $\mathbb{H}^{2} / \Gamma$ is not compact then $\Gamma$ is a free group. If $\mathbb{H}^{2} / \Gamma$ has a cusp then $\delta(\Gamma)>1 / 2$ [1], so again Brooks's result applies. In the remaining case, the result follows from Theorem 1.

\section{References}

1. Beardon, A.: The exponent of convergence of Poincaré series. Proc. Lond. Math. Soc. 18, 461$483(1968)$

2. Brooks, R.: The fundamental group and the spectrum of the Laplacian. Comment. Math. Helv. 56, 581-598 (1981)

3. Brooks, R.: The bottom of the spectrum of a Riemannian covering. J. Reine Angew. Math. 357, 101-114 (1985)

4. Cohen, J.: Cogrowth and amenability in discrete groups. J. Funct. Anal. 48, 301-309 (1982)

5. Ghys, E., de la Harpe, P.: Sur les Groupes Hyperboliques d'après Mikhael Gromov. Progress. in Mathematics, vol. 83. Birkhauser, Boston (1990)

6. Grigorchuk, R.: Symmetrical Random Walks on Discrete Groups Multicomponent Random Systems, Adv Probab Related Topics, vol 6. Dekker pp. 285-325, New York (1980)

7. Hirsch, M., Thurston, W.: Foliated bundles, invariant measures and flat manifolds. Ann. Math. 101, 369-390 (1975)

8. Kaimanovich, V., Vershik, A.: Random walks on discrete groups: boundary and entropy. Ann. Probab. 11, 457-490 (1983)

9. Lalley, S.: Renewal theorems in symbolic dynamics, with applications to geodesic flows, non-Euclidean tessellations and their fractal limits. Acta Math. 163, 1-55 (1989)

10. Ledrappier, F., Sarig, O.: Invariant measures for the horocycle flow on periodic hyperbolic surfaces. to appear, Israel J. Math

11. Ol'shanskii, A.Yu.: On a geometric method in the combinatorial group theory. In: Proceedings of the International Congress of Mathematicians, vol. 1, pp. 415-424(Warsaw, 1983). PWN Warsaw (1984)

12. Ortner, R., Woess, W.: Non-backtracking random walks and cogrowth of graphs. to appear Can. J. Math

13. Pollicott, M., Sharp, R.: Comparison theorems and orbit counting in hyperbolic geometry. Trans. Amr. Math. Soc. 350, 473-499 (1998)

14. Pollicott, M., Sharp, R.: Poincaré Series and Comparison Theorems for Variable Negative Curvature Topology, Ergodic Theory, Real Algebraic Geometry. Amer. Math. Soc. Transl. Ser. 2,vol. 202. American Mathematical Society Providence, RI 2001,pp. 229-240

15. Rees, M.: Checking ergodicity of some geodesic flows with infinite Gibbs measure. Ergodic Theory Dyn. Syst. 1, 107-133 (1981)

16. Roblin, T.: Un théorème de Fatou pour les densités conformes avec applications aux revêtements galoisiens en courbure négative. Israel J. Math. 147, 333-357 (2005)

17. Woess, W.: Cogrowth of groups and simple random walks. Arch. Math. (Basel) 41, 363-370 (1983)

18. Woess, W.: Random Walks on Infinite Graphs and Groups. Cambridge Tracts in Mathematics,vol 138. Cambridge University Press, Cambridge (2000) 